\title{
Counting Distributions and Error Probabilities for Optical Receivers Incorporating Superlattice Avalanche Photodiodes
}

\author{
MALVIN C. TEICH, SENIOR MEMBER, IEEE, KUNIAKI MATSUO, MEMBER, IEEE, \\ AND BAHAA E. A. SALEH, SENIOR MEMBER, IEEE
}

\begin{abstract}
Exact gain distributions and electron counting distributions are presented for superlattice avalanche photodiodes that operate by single-carrier transport perpendicular to the superlattice planes. The characteristic shapes of these distributions are compared with those of the single-carrier conventional avalanche photodiode and the photomultiplier tube. The electron counting distributions, which assume Poisson photocarrier injection, are used to calculate the error performance of a simple optical communication system. This performance is compared with that achievable by a single wcarrier conventional APD receiver of identical quantum efficiency and gain. For simplicity of calculation, the system consists of a transmitter emitting light pulses containing a Poisson number of photons and a maximum-likelihood integrate-and-dump receiver. It makes use of binary on-off keying and is subject to noise events arising from multiplied background radiation and/or multiplied dark noise. The performance of the superlattice photodiode receiver turns out to be always superior to that of the singlecarrier conventional photodiode receiver, for all values of the gain. The advantage can attain several orders of magnitude (even though the excess noise factors for the two devices lie within a factor of two). The superlattice receiver with high impact-ionization probability is shown to behave like an ideal photon counter with the same quantum efficiency, even if the device has many stages. The deleterious effects of receiver thermal noise on probability of error are examined.
\end{abstract}

\section{INTRODUCTION}

A VALANCHE photodiodes (APD's) are important devices, in large part because of their use as detectors in fiber-optic communication systems [1]. They operate by converting clusters of detected photons, associated with information-carrying pulses of light in a digital communication system, into cascades of electrons. These cascades have sufficiently high charge to be readily detected by the electronics following the APD. The multiplication process ${ }^{1}$ introduces noise because the number of electrons

Manuscript received February 4, 1986; revised May 16, 1986. This work was supported by the National Science Foundation and by the Joint Services Electronics Program.

M. C. Teich is with the Center for Telecommunications Research and the Columbia Radiation Laboratory, Department of Electrical Engineering Columbia University, New York, NY 10027.

K. Matsuo was with the Columbia Radiation Laboratory, Department of Electrical Engineering, Columbia University, New York, NY 10027. $\mathrm{He}$ is now with the Hiroshima-Denki Institute of Technology, Hiroshima, Japan.

B. E. A. Saleh is with the Department of Electrical and Computer Engineering, University of Wisconsin, Madison, WI 53706 and with the Columbia Radiation Laboratory, Columbia University, New York, NY 10027.

IEEE Log Number 8609978.

${ }^{2} \mathrm{~A}$ multiplication process is also referred to as a cascade process, a branching process, and a gain process. created per detected photon varies from trial to trial. Nevertheless, this noise is tolerated as long as it is small in comparison with the additive thermal noise of the amplifiers and other electronics following the APD.

One way of characterizing multiplicative noise in a photodetector is by means of the excess noise factor $F_{e}$. Expressions for $F_{e}$ have recently been presented in uniform notation [2] for several kinds of photodetectors: the double-carrier conventional avalanche photodiode (CAPD), the double-carrier superlattice avalanche photodiode (SAPD), and the photomultiplier tube (PMT). In all cases, the multiplication was assumed to be instantaneous. $^{2}$

The noisiness of both the CAPD and the SAPD is minimized under single-carrier-initiated/single-carrier multiplication (SCISCM) conditions. ${ }^{3}$ The excess noise factors for both devices then lie below 2 for all values of the average multiplication $\langle M\rangle$. This can be seen quite clearly in the graphical representation provided in [2, Fig. 3]. Although the theoretical excess noise factor for the SAPD is always lower than that of the CAPD [2], the differential is never very large since $F_{e}<2$ for both cases. The excess noise factor for a high-gain first-dynode (GaP) PMT, with Poisson secondary-emission multiplication, is also presented in [2, Fig. 3]. ${ }^{4} F_{e}$ for the PMT can fall below that for the SAPD, but, again, the range is restricted to 1 $\leq F_{e}<2$.

CAPD's with high quantum efficiencies and near-ideal noise behavior have, in fact, been fabricated. At RCA, Si devices have been made that operate in the wavelength region from 0.4 to $0.95 \mu \mathrm{m}$, with quantum efficiencies $\eta$ $\approx 0.8$. Some of these exhibit excess noise factors as low as $F_{e}=2.6$, corresponding to $k_{c}$ (ratio of hole- to elec-

${ }^{2}$ Instantaneous multiplication means that the detector integration time is sufficiently long so that the entire current pulse is captured as a charge.

${ }^{3}$ The designation SCISCM means that a only single kind of carrier (viz., either electrons or holes) initiates the avalanche process and that only this kind of carrier creates new carrier pairs by impact ionization. This is to be distinguished from the designation "single injected electron," used later, which means that only one electron is injected.

${ }^{4}$ In the example presented in [2, Fig. 3], it is assumed that all dynodes of the PMT have Poisson secondary-emission multiplication and that the first dynode has a gain that is 10 times as large as that of the following dynodes $(A=10)$. The average multiplication of the one- and four-stage ( $m=1,4)$ GaP PMT is approximately the same as that of the five- and ten-stage ( $m=5,10$ ) SAPD, respectively. 
tron-ionization coefficients) $=0.006$ at $\langle M\rangle=1003$ ] Even quieter devices, with $F_{e}<2.2$ corresponding to $k_{c}$ $\langle 0.002$ at $\langle M\rangle=100$, are possible [4]. Si CAPD devices with essentially SCISCM properties are therefore currently available for use at wavelengths below abou t. 1 $\mu \mathrm{m}$.

However, in the wavelength region $\lambda \approx 1.3$ to $1.6 \mu \mathrm{m}$, which is of interest for fiber-optic communications [1] $\mathrm{Si}$ is inadequate and CAPD's are generally fabricated from quaternary III-V materials. Unfortunately $k_{c} \approx 1$ in these materials so that $F_{e}$ is substantially greater than 2 [5]. Dark current and leakage current may also present difficulties in such devices [5].

As a result, there have recently been a number of proposals suggesting the use of novel heterostructure devices that reduce tunneling currents and enhance the ionizationcoefficient ratio (i.e., render the multiplication more single-carrier-like) [5], [6]. This would open the way to sm all high-quantum efficiency low-voltage low-noise detectiors in the near infrared. The first such proposal, by Chin et al. [7], suggested the use of a multiquantum-well superlattice avalanche photodiode (SAPD) consisting of an alternating series of wide- and narrow-bandgap layers. It has been shown both experimentally [8]-[10], and by means of many-particle Monte Carlo simulations [1!], [12], that such a structure can indeed provide an enhanced ionization ratio. Since the carrier transport is perpend $c-$ ular to the planes of the superlattice, the carriers gain $\mathrm{n}$ ergy at the heterointerface potential discontinuity at each period of the multilayer structure.

Other superlattice configurations that have been cons ilered include the graded-gap staircase SAPD devised oy Williams, Capasso, and Tsang [13]-[15] and investigated by Matsuo et al. [16]; the doped-quantum-well SAF'D conceived by Blauvelt et al. [17] and studied by Brennan [18]; and the stored-carrier multiquantum-well SAPD of Smith et al. [19] (see also Chuang and Hess [20] and Calpasso et al. [21]). There is currently an effort underway at AT\&T Bell Laboratories to fabricate a staircase SAI D for operation in this longer wavelength region [22]. However, residual hole ionization can be a serious source of unwanted noise in all of these structures and it is imptative to minimize this effect [2], [11], [12], [18].

Although PMT's are precluded by their large size fron candidacy as components in modern fiber-optic systems, their excellent noise characteristics serve as a benchmak for other detectors. It is for this reason that a discussion of their properties is included here. Other desirable pro serties exhibited by PMT's include low dark current, his h gain, good pulse resolution, and ease of operation in the photon-counting mode. On the negative side, aside fro $n$ their large size, are limited quantum efficiency, high-voltage requirements, luminescence noise, and the presence of afterpulsing due to $\mathrm{H}^{+}$ions or inverse photoemissic n [23].

Although the excess noise factor is a useful statistic for characterizing the noisiness of detectors, it has its limitations. It represents, in a compact way, the lowest ordert statistical properties of the gain fluctuations introduced by the multiplication process. However, it does not provide a complete statistical description of the electron current. Although it is useful for the calculation of quantities such as the signal-to-noise ratio (SNR) for analog detection, in general it is inadequate for describing the performance of a digital-signal information transmission system [24], [25]. Appropriate measures for the performance of such systems are probability of error and probability of detection. These quantities require a more complete statistical description of the electron current, such as the electron counting distribution. Indeed, these performance measures are especially dependent on the tails of the counting distributions which are generally only weakly reflected in the excess noise factor. Certain photodetectors will have more favorable shapes for minimizing error probabilities than will others.

The purpose of this paper is twofold. First we calculate, and graphically display, representative exact gain distributions (single-electron injection) and electron counting distributions (Poisson electron injection) for single-carrier CAPD's, single-carrier SAPD's, and PMT's. The characteristic shapes of these distributions are compared and contrasted for the three detectors. Second, we use the electron counting distributions to calculate the probability of error achievable by a simple digital optical communication system. The performance of optical receivers incorporating CAPD's and SAPD's with the lowest possible noise (i.e., single-carrier devices) are compared. For simplicity of calculation, the system is assumed to consist of a transmitter emitting light pulses containing a Poisson number of photons (e.g., a laser or LED) and a maximum-likelihood optical receiver [26], [27]. It makes use of binary on-off keying (OOK) and is subject to noise events that are assumed to arise from multiplied background radiation and/or multiplied dark noise [27]. A1though inter-symbol interference, fiber noise, and detector $1 / f$ noise are all assumed negligible, the deleterious effects of receiver thermal noise on performance are examined for these two detectors.

\section{Detector Electron Counting Statistics}

Consider a point process representing the primary (photon-generated) carriers. Let the number of these carriers generated within the time interval $[0, T]$ be described by the discrete random variable $a$. Let each of these primary carriers, in turn, independently produce $M$ secondary (or daughter) carriers. $M$ is the discrete gain random variable representing the carrier multiplication. The total number of electrons $n$ produced at the output of the device in $[0$, $T]$ is the quantity of interest.

If $a$ and $M$ are statistically independent, which is safe to assume, then the overall electron count mean and variance are given by [2], [23], [28]-[31]

$$
\langle n\rangle=\langle M\rangle\langle a\rangle
$$

and

$$
\operatorname{Var}(n)=\langle M\rangle^{2} \operatorname{Var}(a)+\langle a\rangle \operatorname{Var}(M)
$$


respectively. The angular brackets $\langle\cdot\rangle$ represent ensemble averages. Equation (2) can be written as [2]

$$
\operatorname{Var}(n)=\langle a\rangle\langle M\rangle^{2}\left[\mathcal{F}_{a}+\left(F_{e}-1\right)\right]
$$

where $F_{e}$ represents the excess noise factor for the detector

$$
F_{e} \equiv\left\langle M^{2}\right\rangle /\langle M\rangle^{2}
$$

and $\mathfrak{F}_{a}$ represents the Fano factor for the photogenerated carriers,

$$
F_{a}=\operatorname{Var}(a) /\langle a\rangle .
$$

When the device is illuminated by Poisson photons, $\mathfrak{F}_{a}=$ 1, whereupon (3) reduces to the familiar expression [2]

$$
\operatorname{Var}(n)=\langle a\rangle\langle M\rangle^{2} F_{e} .
$$

The principal interest here is the entire electron counting distribution $q(n)$, rather than just its mean and variance as described above. This is most easily obtained via the moment generation functions (mgf's) [32]

$$
Q_{x}(s)=\langle\exp (-s x)\rangle
$$

for the counting processes $x=a, M$, and $n$. The general relationship among the mgf's under cascading is well known [30]-[33]

$$
Q_{n}(s)=Q_{a}\left[-\ln Q_{M}(s)\right] .
$$

Under Poisson illumination, the mgf for the Poisson photocarriers (primary events) is [32], [33]

$$
Q_{a}(s)=\exp \left[\langle a\rangle\left(e^{-s}-1\right)\right],
$$

whereupon (8) becomes [32], [33]

$$
Q_{n}(s)=\exp \left[\langle a\rangle\left(Q_{M}(s)-1\right)\right] .
$$

Equation (10) represents the moment generating function for a so-called generalized Poisson distribution [32]. The count mean and variance, (1) and (2), follow immediately by differentiation [32].

The electron counting distribution $q(n)$ is contained in (10). The most direct way to obtain it is by replacing $e^{-s}$ by $z$, which converts $(10)$ to a probability generating function (pgf) $G_{n}(z)$, i.e.,

$$
G_{n}(z)=\left\langle z^{n}\right\rangle=Q_{n}(s=-\ln z) .
$$

The electron counting probabilities $q(n)$ may then be obtained from the pgf by means of the formula [34]

$$
q(n)=\left.\frac{1}{n !} \frac{\partial^{n}}{\partial z^{n}} G_{n}(z)\right|_{z=0} .
$$

The gain distribution $p(M)$, representing the response to a single injected electron, ${ }^{3}$ is obtained in the same way but $Q_{M}(s)$ and $G_{M}(z)$ are used rather than (10) and (11) [16].

In the following sections, we present formulas for the electron counting distributions for the single-carrier CAPD, single-carrier SAPD, and PMT, respectively. The gain mgf $Q_{M}(s)$, representing the response to a single in- jected electron, is, of course, different for each case. To distinguish the results, all quantities are labeled with the designations $y, m$, and $u$ for the three detectors, respectively. It is assumed that absorption takes place outside the multiplication region. Instantaneous multiplication is assumed in all cases. ${ }^{2}$

\section{Counting Statistics for the Single-Carrier Conventional APD}

The gain distribution for the single-carrier CAPD, $p_{y}(M)$, is obtained from the Yule-Furry birth process [2], [32], [34]-[36]..$^{5}$ The result is the shifted Bose-Einstein distribution, as was established independently by Personick [33, Eq. (25)] and McIntyre [37, Eq. (16)]. McIntyre also showed that, given an arbitrary (deterministic) number of photocarriers, the increase in the electron population is described by the negative-binomial distribution. This is in accord with well-known results for the YuleFurry process [32]. [33]

The shifted Bose-Einstein probability distribution is

$$
p_{y}(M)= \begin{cases}\frac{1}{\left\langle M_{y}\right\rangle}\left(\frac{\left\langle M_{y}\right\rangle-1}{\left\langle M_{y}\right\rangle}\right)^{M-1}, & M=1,2, \cdots \\ 0, & M=0\end{cases}
$$

where the mean electron number (average gain) $\left\langle M_{y}\right\rangle$ is

$$
\left\langle M_{y}\right\rangle=\exp (y)
$$

and the variance is

$$
\operatorname{Var}\left(M_{y}\right)=\exp (y)[\exp (y)-1] .
$$

This distribution is plotted versus the gain random variable $M$ in Fig. 1 for $y=0.55,1.76$, and 2, corresponding to $\left\langle M_{y}\right\rangle=1.73,5.83$, and 7.41 , respectively. The distributions are monotonic decreasing and become progressively broader as $y$ increases. The excess noise factor, obtained by using (4), (13b), and (13c), is

$$
F_{e}=2-\exp (-y) .
$$

If the number of initial photocarriers is instead Poisson, the electron counting distribution $q_{y}(n)$ is the Poissondriven shifted-Bose-Einstein distribution [34], [33, Eq. (35)]. Its statistical properties follow from the mgf of the Yule-Furry process with a Poisson initial population $Q_{n}(y, s)[34$, Eq. (24)]

$$
Q_{n}(y, s)=\exp \left\{-\langle a\rangle \frac{1-\exp (-s)}{1-[1-\exp (-y)] \exp (-s)}\right\} .
$$

\footnotetext{
${ }^{5}$ Yule [35] was concerned with the growth of species in some genus of animals or plants. Furry [36] considered the development of an idealized cosmic-ray shower from a single initial particle.
} 
CAPD: SINGLE INJECTED ELECTRON
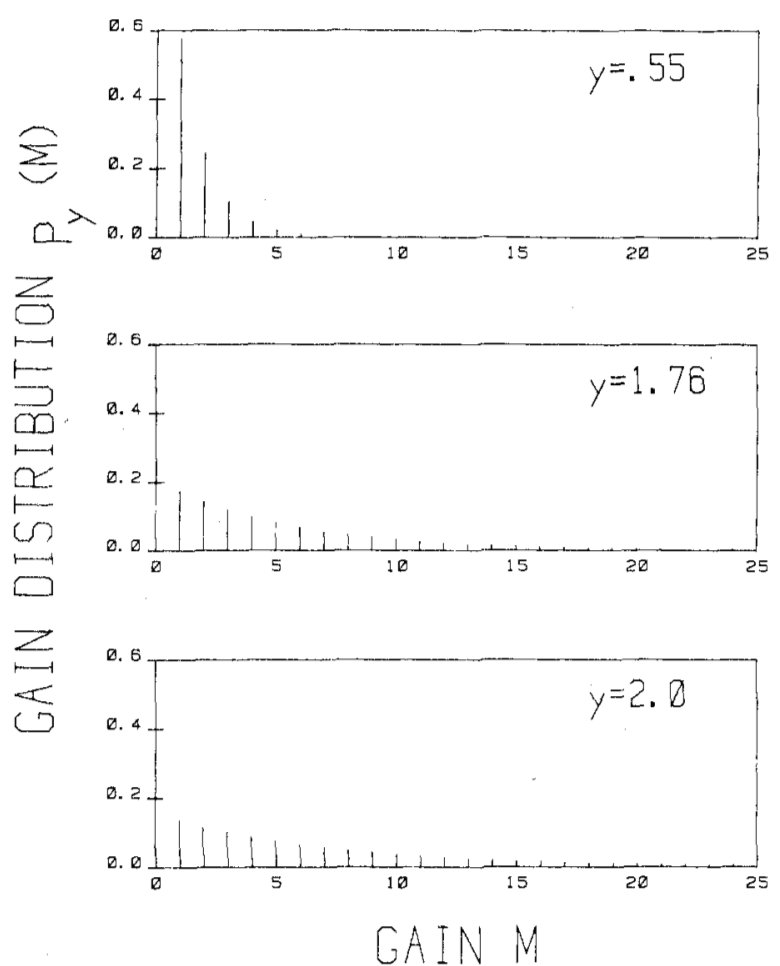

Fig. 1. Gain distribution $p_{y}(M)$ versus gain $M$ for the single-carrier-initiated/single-carrier multiplication CAPD. This is the electron counling distribution in response to a single detected photon. The characteritic branching parameter of the device is denoted $y$; the average gain is $\langle 11$, $=\exp (y)$. Theoretical gain distributions are shown for $y=0.55,1.75$, and 2 , corresponding to average gains of $1.73,5.83$, and 7.41 , resp 30 tively. The (shifted Bose-Einstein) distributions, which are monoto lic decreasing, become progressively broader as $y$ increases.

The quantity $n$ represents the number of electrons at the output of the device; $\langle a\rangle$ is the average number of firimary Poisson photocarriers, and $y$ is the characteris c branching parameter associated with the Yule-Furry $m i l-$ tiplication process in the detector (i.e., the product of the impact-ionization probability $\alpha$ or $\beta$, and the width of the depletion region $w[2])$.

The electron count mean and variance may be obtain ad in several ways: by direct differentiation of (14), from (1) and (2) using (13b) and (13c), or from [34]. They are

$$
\left\langle n_{y}\right\rangle=\langle a\rangle \exp (y)
$$

and

$$
\operatorname{Var}\left(n_{y}\right)=\langle a\rangle \exp (y)[2 \exp (y)-1]
$$

respectively.

The probability $q_{y}(n)$ of observing $n$ electrons at the detector output follows from (14) with the help of (11) and (12), or directly from [34, Eq. (28)]. It is writt:n most directly in terms of the recursion relation

$$
\begin{aligned}
q_{y}(n+1) & =\frac{\left\langle n_{y}\right\rangle}{n+1} \sum_{k=0}^{n} q_{y}(n-k) J_{y}(k), \\
n=0,1,2, \cdots & \\
q_{y}(0)= & \exp (-\langle a\rangle)
\end{aligned}
$$

CAPD: POISSON INJECTEO ELECTRONS, $<a>=1$
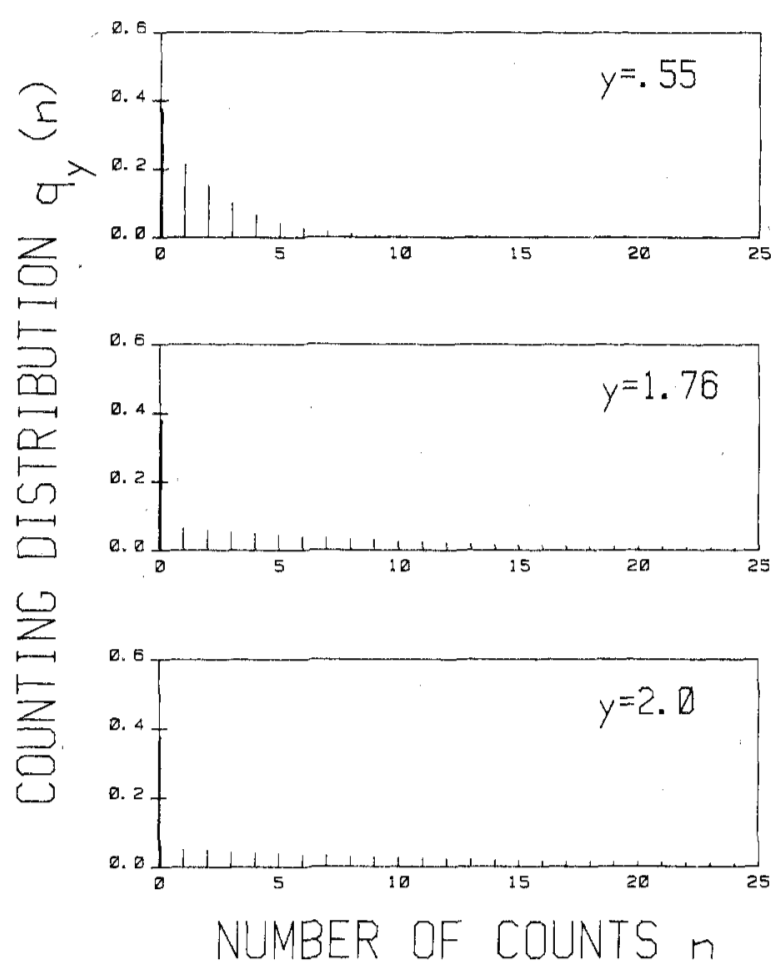

Fig. 2. Electron counting distribution $q_{y}(n)$ versus number of electrons $n$ for the SCISCM CAPD, in response to a Poisson-distributed number of injected electrons with mean value $\langle a\rangle=1$. Theoretical counting distributions are shown for $y=0.55,1.76$, and 2 , corresponding to average gains of $1.73,5.83$, and 7.41 , respectively. The results differ from those illustrated in Fig. 1 for a single injected electron, although the means are identical. The detection of a Poisson number of photons causes the counting distributions to become a weighted repetition of the distributions shown in Fig. 1. The registration of zero events becomes possible and the variance of the distribution is substantially increased.

where

$$
J_{y}(k)=(k+1) \exp (-2 y)[1-\exp (-y)]^{k} .
$$

This distribution is shown in Fig. 2, for $y=0.55,1.76$, and 2 , corresponding to average gains of $1.73,5.83$, and 7.41 , respectively, since the mean number of Poisson injected electrons has been chosen to be $\langle a\rangle=1$. The distributions $q_{y}(n)$ differ substantially from those illustrated in Fig. 1 for a single injected electron $p_{y}(n)$, even though the means are identical $\left(\left\langle n_{y}\right\rangle=\langle a\rangle\left\langle M_{y}\right\rangle=\left\langle M_{y}\right\rangle\right)$. The detection of a Poisson number of photons makes it possible to register zero events and also broadens the distributions considerably. Webb et al. [24, Eq. (20)] have provided an approximation to (16) under certain restrictions.

To provide a basis for comparison with the SAPD (for which double-carrier distributions are not available), only SCISCM electron counting distributions have been presented for the CAPD. More general electron counting distributions, applicable for a CAPD with Poisson initial population and double-carrier multiplication, are available. These have been obtained by Personick [38], Conradi [39], and in approximate form by Webb et al. [24, 


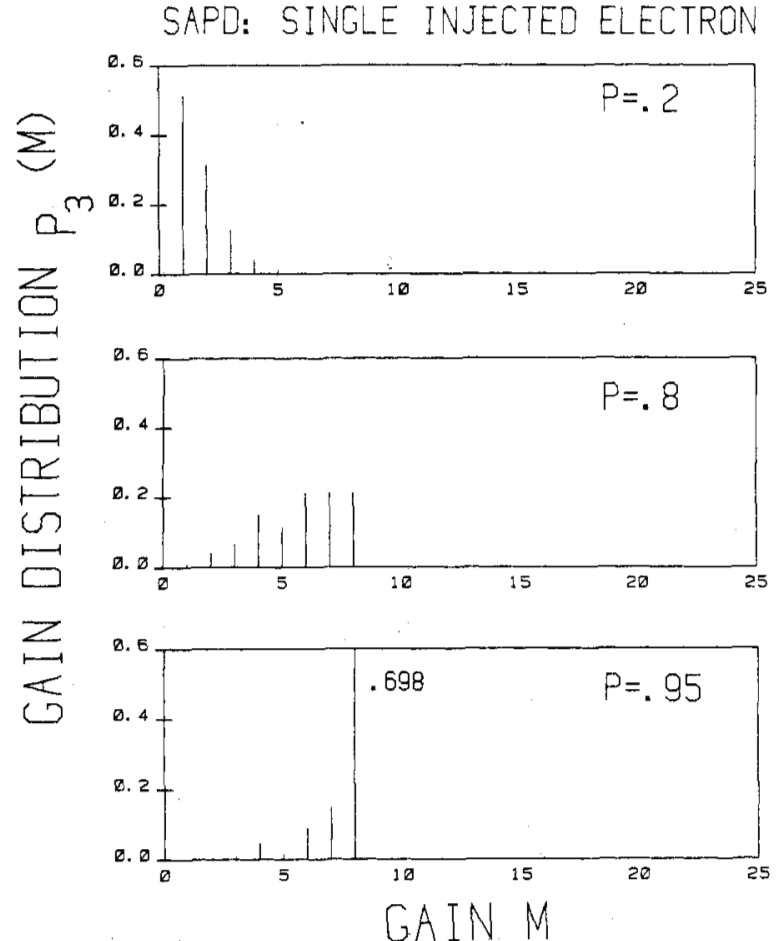

Fig. 3. Gain distribution $p_{m}(M)$ versus gain $M$ for the single-carrier SAPD This is the electron counting distribution in response to a single detected photon. The parameter $m$ is the number of stages in the device and $P$ is the electron impact-ionization probability. Theoretical gain distributions are shown for $m=3$ with $P=0.2,0.8$, and 0.95 , corresponding to average gains of $1.73,5.83$, and 7.41 , respectively. Nonmonotonicities in the distributions reflect failures to multiply early in the chain. The average gain is $\left\langle M_{m}\right\rangle=(1+P) m$; it is limited to a maximum value of $2^{m}$. Higher values of the gain random variable achieve greater probabilities as $P$ increases. As $P$ approaches unity, the multiple peaks disappear and the distribution narrows to a delta function at $M=2^{m}$. The results presented here are decidedly different from those for the CAPD shown in Fig. 1 (after Matsuo et al. [16, Fig. 4]).

Eq. (20)] ${ }^{6}$ A method for calculating the tail probabilities with arbitrary accuracy has recently been provided by Helstrom [41].

\section{Counting Statistics for the Single-Carrier SUPERLATTICE APD}

Matsuo et al. [16] recently derived the gain distribution $p_{m}(M)$ for a single-carrier instantaneous-multiplication SAPD in terms of the number of stages of the device $m$ and the impact-ionization probability per stage $P$. Although the analysis was explicitly presented in terms of the graded-gap staircase SAPD, it is in fact applicable for any SAPD in which the carrier transport takes place perpendicular to the superlattice planes. ${ }^{7}$ The result is given

${ }^{6}$ Similar stochastic counting processes have been dealt with in the context of cosmic-ray cascades where high-energy electrons and gamma rays play the roles of electrons and holes in the APD, respectively [40].

The results in [16] were derived for a single injected electron. What is called the gain distribution $p_{m}(M)$ in this paper (where $M$ is the gain random variable) was called the electron counting distribution $p_{m}(n)$ (with random variable $N_{m}$ ) in [16]. Since the analysis in [16] is valid only for perpendicular-carrier-transport SAPD's, it will not apply to the channeling APD [42], [43], nor to other devices in which the carriers are spatially separated by means of a transverse field with transport taking place in the plane of the layers.
SAPD: POISSON INJECTED ELECTRONS, $<a>=1$
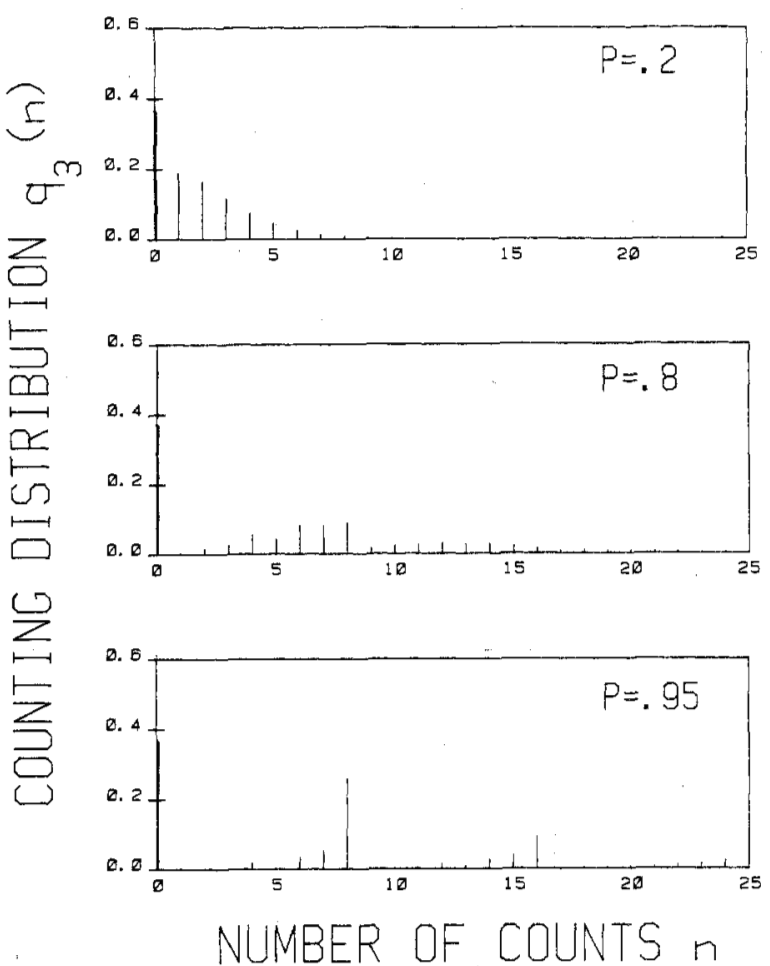

Fig. 4. Electron counting distribution $q_{m}(n)$ versus $n$ for the single-carrier SAPD, in response to a Poisson-distributed number of injected electrons with mean value $\langle a\rangle=1$. Theoretical counting distributions are shown for $m=3$ with $P=0.2,0.8$, and 0.95 , corresponding to average gains of $1.73,5.83$, and 7.41 , respectively. The results are quite different from those illustrated in Fig. 3 for a single-injected electron, although the means are identical. The detection of a Poisson number of photons causes the counting distribution to become a weighted repetition of the distributions shown in Fig. 3. This is most evident for $P=0.95$. The registration of zero events becomes possible and the variance of the distribution is substantially increased. The maximum allowed count number is no longer restricted to $2^{m}$.

by the recursion relation [16, Eq. (12)]

$$
\begin{aligned}
p_{m}(M)= & (1-P) p_{m-1}(M) \\
& +P \sum_{k=0}^{M} p_{m-1}(M-k) p_{m-1}(k), \\
& M \geq 1, m \geq 1 \\
p_{m}(0)= & 0, \quad m \geq 1 \\
p_{0}(M)= & \delta_{1, M} .
\end{aligned}
$$

The mean and variance of the gain are

$$
\left\langle M_{m}\right\rangle=(1+P)^{m}
$$

and

$$
\operatorname{Var}\left(M_{m}\right)=(1-P)\left[(1+P)^{2 m-1}-(1+P)^{m-1}\right]
$$

respectively. This distribution is shown in Fig. 3 for a three-stage device $(m=3)$ with $P=0.2,0.8$, and 0.95 . These correspond to average gains of $1.73,5.83$, and 7.41 , respectively. Nonmonotonicities in the distributions 
reflect failures to multiply early in the chain [16]. Higher values of the gain random variable achieve greater probabilities as $P$ increases. As $P$ approaches unity, the rultiple peaks disappear and the distribution narrows to a delta function at the maximum allowed gain $M=2^{3}$. These results are decidedly different from those for the CAPD shown in Fig. 1, which continue to broaden as $y$ increases. The narrowing reflects one of the potential benefits of the SAPD. The excess noise factor is obtained with the help of (4), (17b), and (17c)

$$
F_{e}=1+[(1-P) /(1+P)]\left[1-(1+P)^{-m}\right] \text {. }
$$

If the number of initial photocarriers is instead Poisson, the moment generating function for the electron count $Q_{n}(m, s)$ follows from (10) together with the recursion relation for $Q_{M}(m, s)$, which is given by [16, Eq. (4)]

$$
\begin{aligned}
Q_{M}(m, s)= & (1-P) Q_{M}(m-1, s) \\
& +P\left[Q_{M}(m-1, s)\right]^{2}, \quad m \geq 1 \\
Q_{M}(0, s)= & e^{-s} .
\end{aligned}
$$

The electron count mean $\left\langle n_{m}\right\rangle$ and variance $\operatorname{Var}\left(r_{m}\right)$ are most readily obtained from (1), (2), (17b), and (17c); they are

$$
\left\langle n_{m}\right\rangle=\langle a\rangle(1+P)^{m}
$$

and

$$
\begin{aligned}
\operatorname{Var}\left(n_{m}\right)= & \langle a\rangle\left\{(1+3 P)(1+P)^{m-1}\right. \\
& \left.+2(1+P)^{m}\left[(1+P)^{m-1}-1\right]\right\}
\end{aligned}
$$

respectively.

Finally, using (10)-(12) and (18) we obtain the electron counting distribution $q_{m}(n)$ at the output of the SAPD, in response to a Poisson number of photocarriers at the input

$$
\begin{aligned}
q_{m}(0) & =\exp (-\langle a\rangle) \\
q_{m}(n+1) & =\frac{\langle a\rangle}{n+1} \sum_{k=0}^{n}(k+1) q_{m}(n-k) A_{m}(k+1)
\end{aligned}
$$

where

$$
\begin{aligned}
A_{m}(k)= & (1-P) A_{m-1}(k) \\
& +P \sum_{j=1}^{k-1} A_{m-1}(k-j) A_{m-1}(j), \\
& \quad k \neq 0, m=1,2,3, \cdots \\
A_{m}(0)= & 0, \quad \text { for all } m \\
A_{0}(1)= & 1 .
\end{aligned}
$$

This distribution is presented in Fig. 4 for a three-stage SAPD $(m=3)$ with $P=0.2,0.8$, and 0.95 , and $\langle a\rangle=$ 1 , corresponding to average gains of $1.73,5.83$, and 7.41 , respectively. The results differ substantially from those illustrated in Fig. 3 for a single injected electron, al- though the means are identical $\left(\left\langle n_{3}\right\rangle=\langle a\rangle\left\langle M_{3}\right\rangle=\right.$ $\left.\left\langle M_{3}\right\rangle\right)$. The detection of a Poisson number of photons causes the counting distribution to become a weighted repetition of the distributions shown in Fig. 3. This is most evident for $P=0.95$. The registration of zero events becomes possible and the variance of the distribution is substantially increased. The maximum allowed count number is no longer restricted to $2^{3}$.

When $P$ is sufficiently large, the character of $q_{m}(n)$ illustrated in Fig. 4 is distinctly different from the character of $q_{y}(n)$ for the CAPD illustrated in Fig. 2, even though the means are again identical. This reflects the more deterministic character of the multiplication process in the SAPD. It is also worthy of mention that $q_{m}(n)$ is distinctly non-Gaussian and remains so for arbitrarily large mean count number. Indeed as $P \rightarrow 1, q_{m}(n)$ becomes the fixedmultiplicative Poisson distribution, which is highly scalloped [44]. As will become evident in the sequel, this characteristic admits the possibility of improved system performance. When $P$ is small, the SAPD results are not dissimilar from those of the CAPD, for reasons that have been discussed elsewhere [2].

\section{Counting Statistics for the PMT}

For comparison purposes, we also present the counting statistics at the output of a $u$-stage PMT where all dynodes have identical Poisson secondary-emission statistics with average multiplication $\delta$. The moment generating function for the gain $Q_{M}(u, s)$ is obtained by successive generalization of the Poisson mgf with itself, i.e., [45, Eq. $(2)]^{8}$

$$
\begin{array}{ll}
Q_{M}(u, s)=Q_{M}\left(1,-\ln Q_{M}(u-1, s)\right), & u>1 \\
Q_{M}(1, s)=\exp \{\delta[\exp (-s)-1]\}, & u=1 \\
Q_{M}(0, s)=\exp (-s) . &
\end{array}
$$

The probability distribution of the gain $p_{u}(M)$, along with its mean and variance, can be found in a variety of sources [45]-[48]. The excess noise factor is also well known [23], [2, Eq. (44) with $A=1$ ].

The gain statistics for a single-stage $(u=1)$ PMT, $p_{1}(M)$, are illustrated in Fig. 5 for $\delta=1.73,5.83$, and 7.41. This represents the Poisson secondary-emission

$$
\begin{aligned}
& { }^{8} \text { Equation (2) in [45] should read } \\
& Q_{m}(s)=Q_{1}\left(-\ln Q_{m-1}(s)\right) \\
& \underbrace{Q_{m}(s)=Q_{1}\left(-\ln Q_{1}\left(-\ln Q_{1}\left(-\ln Q_{1} \cdots-\ln Q_{1}(s)\right)\right)\right)}_{m \text { times }} \\
& Q_{0}(s)=\exp (-s) \\
& Q_{1}(s)=\exp \{a[\exp (-s)-1]\} \text {. }
\end{aligned}
$$

The error was in transcription; all calculations and results in [45] are correct. In this paper $u$ plays the role of $m$. 
PMT: SINGLE PHOTOELECTRON
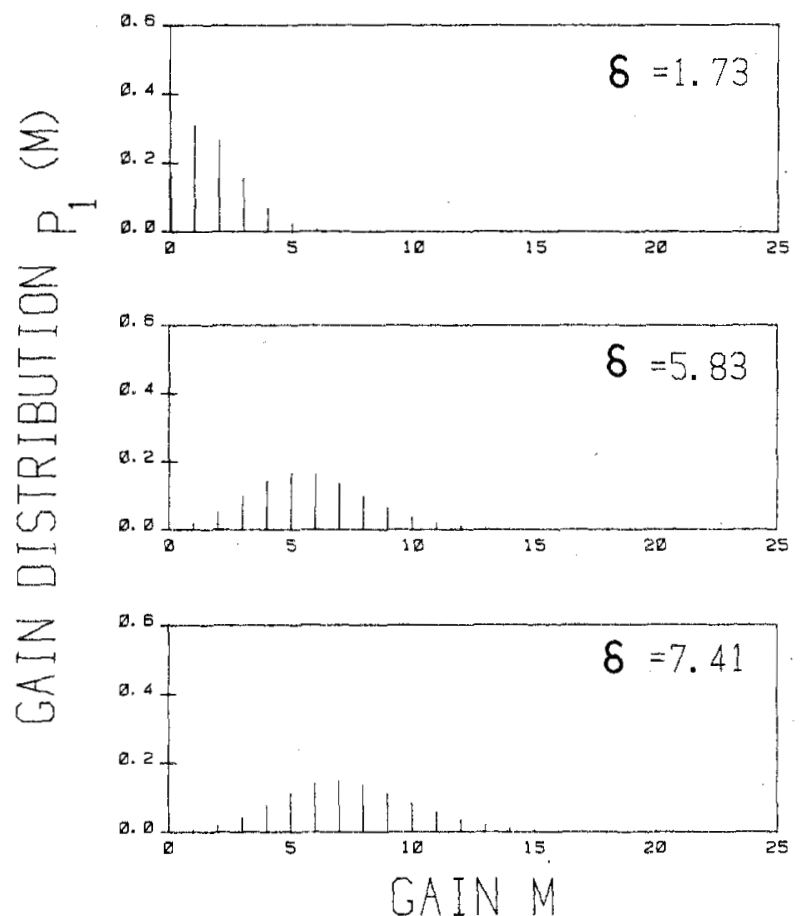

Fig. 5. Gain distribution $p_{u}(M)$ versus gain $M$ for the single-stage PMT with Poisson multiplication. This represents the secondary-emission electron counting distribution in response to a single detected photon. The parameter $u$ is the number of PMT stages and $\delta$ is the average number of secondary-emission electrons per photoelectron. Theoretical Poisson gain distributions are shown for $\delta=1.73,5.83$, and 7.41. There is a finite probability of observing zero secondary-emission electrons resulting from the failure of the dynode to respond to the photoelectron. The character of the gain distribution is rather intermediate between that of the CAPD (Fig. 1) and the SAPD (Fig. 3).

electron counting distribution in response to a single photoelectron. Results for the single-stage PMT are illustrated because the average gain values can realistically be made the same as those of the CAPD and SAPD for comparison purposes. Unlike the gain distributions for the CAPD and SAPD, there is a finite probability that secondary emission will result in zero output electrons. The character of the gain distribution is rather intermediate between that of the CAPD (Fig. 1) and the SAPD (Fig. 3).

For a Poisson number of initial photoelectrons, the moment generating function for the number of electrons at the output of the PMT, $Q_{n}(u, s)$, is obtained by the use of (10) and (22). Since the secondary-emission multiplications are assumed to be Poisson, the result is simply an additional generalization of (22a) with one more Poisson moment generating function.

The mean $\left\langle n_{u}\right\rangle$ and variance $\operatorname{Var}\left(n_{u}\right)$ of the output electron count are readily obtained [23], [45]. For identical dynodes, these quantities turn out to be

$$
\left\langle n_{u}\right\rangle=\langle a\rangle \delta^{u}
$$

and

$$
\operatorname{Var}\left(n_{u}\right)=\langle a\rangle \delta^{u}\left[1+\delta\left(1-\delta^{u}\right) /(1-\delta)\right]
$$

respectively.

$$
\text { PMT: POISSON PHOTOELECTRONS, }<a>=1
$$
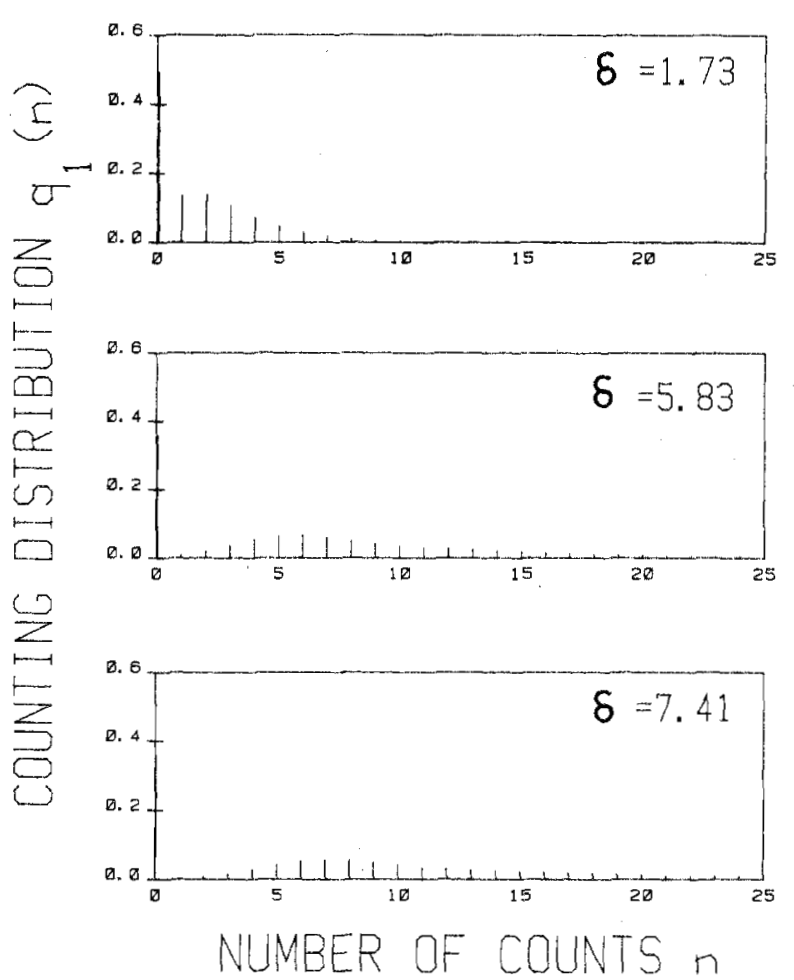

Fig. 6. Electron counting distribution $q_{u}(n)$ versus number of electrons $n$ for the single-stage PMT with Poisson secondary-emission multiplication, in response to a Poisson-distributed number of injected electrons with mean value $\langle a\rangle=1$. The theoretical (Neyman type-A) counting distributions are shown for $\delta=1.73,5.83$, and 7.41 . The character of this distribution is somewhat intermediate between that of the Poissondriven CAPD (Fig, 2) and the Poisson-driven SAPD (Fig. 4); some evidence of nonmonotonicity can be discerned.

Finally, the electron counting distribution at the output, in response to a Poisson number of carriers at the input $q_{u}(n)$ is given by the recursion relation in [45, Eqs. (16) and (17)]. For a one-stage PMT, the result is the wellknown Neyman type-A distribution [44], [49]. This is presented in Fig. 6 for $\langle a\rangle=1$. Theoretical counting distributions are shown for $\left\langle n_{1}\right\rangle=1.73,5.83$, and 7.41. Again these distributions differ from those shown in Fig. 5 even though the means are identical, much as Figs. 4 and 2 differ from Figs. 3 and 1, respectively. The character of these distributions are also somewhat intermediate between those of the Poisson-driven CAPD (Fig. 2) and the Poisson-driven SAPD (Fig. 4); some evidence of nonmonotonicity can be discerned. In the limit as $\langle a\rangle$ increases with $\delta$ held fixed, it can be shown [44] that the Neyman type-A converges in distribution to the Gaussian with mean $\left\langle n_{1}\right\rangle=\langle a\rangle \delta$ and $\operatorname{Var}\left(n_{1}\right)=\langle a\rangle \delta(1+\delta)$.

\section{Comparison of CAPD and SAPD Optical Receiver Performance in the Multiplication- NOISE-LIMITED REgIME}

The electron counting distributions for the single-carrier CAPD and SAPD are now used to numerically calculate error probabilities for the simple maximum-likeli- 
hood integrate-and-dump optical receiver. This will permit a performance comparison to be made between systems incorporating CAPD's and SAPD's in the multiplication-noise-limited domain. Operation in this regime is achieved when the gain is sufficiently high to overcome preamplifier thermal noise.

Let hypotheses $H_{0}$ and $H_{1}$ represent the absence of signal (noise alone), and the presence of signal (noise-plussignal), respectively. The noise events, as well as the signal, are assumed to undergo random multiplication in the APD. Under these hypotheses, the mean number of Poisson injected photoelectrons per pulse are taken to be $\left\langle c_{0}\right\rangle$ and $\left\langle a_{1}\right\rangle$, and the conditional count probabilities are taken to be $q\left(n \mid H_{0}\right)$ and $q\left(n \mid H_{1}\right)$, respectively. Equations (16) and (21) provide the counting distributions $q_{y}(n)$ and $q_{m}(n)$ for the CAPD and SAPD, respectively.

To forge a reasonable comparison between the two :eceivers, the quantum efficiencies $\eta$ and mean gains $\langle M\rangle$ of the CAPD and SAPD are taken to be identical. The identity of the quantum efficiencies provides that the sarne mean numbers of photons per pulse fall on the devices under the two hypotheses (i.e., $\left\langle a_{0}\right\rangle / \eta$ and $\left\langle a_{1}\right\rangle / \eta$ uncier $H_{0}$ and $H_{1}$, respectively). Equality of the mean gains is provided by equating (13b) and (17b), which gives

$$
e^{y}=(1+P)^{m} \text {. }
$$

In maximum-likelihood detection the observation space $Z$ is divided into two parts, $Z_{0}$ and $Z_{1}$, in accordance with

$$
\begin{aligned}
& Z_{0}=\left\{n \mid q\left(n \mid H_{0}\right) \geq q\left(n \mid H_{1}\right)\right\} \\
& Z_{1}=\left\{n \mid q\left(n \mid H_{0}\right)<q\left(n \mid H_{1}\right)\right\} .
\end{aligned}
$$

When an observation falls in $Z_{0}$, we say $H_{0}$; when it falls in $Z_{1}$, we say $H_{1}$. The error probability $P_{e}$ is then

$$
P_{e}=\frac{1}{2}\left(P_{F}+P_{M}\right)
$$

where the false-alarm and miss probabilities are given by

$$
P_{F}=\sum_{Z_{1}} q\left(n \mid H_{0}\right)
$$

and

$$
P_{M}=\sum_{Z_{0}} q\left(n \mid H_{1}\right)
$$

respectively.

System performance is determined by the error probability $P_{e}$, as specified in (27)-(29). In Figs. 7 and 8 wee plot $P_{e}$ versus the Poisson driving (signal-to-noise) rat o $\left\langle a_{1}\right\rangle\left\langle\left\langle a_{0}\right\rangle\right.$ for the CAPD (dashed curves) and SAPD (dotted curves) with several sets of parameters. ${ }^{9}$ The mec.n number of injected photoelectrons per pulse under hypothesis $H_{0}$ was chosen to be exactly $\left\langle a_{0}\right\rangle=1.0$ for all cases. Thus, Figs. 2 and 4 represent precisely the noise

\footnotetext{
${ }^{9}$ The Poisson driving ratio $\left\langle a_{1}\right\rangle /\left\langle a_{0}\right\rangle$ is sometimes specified in terms of the extinction ratio [1] $E=\left\langle a_{0}\right\rangle /\left\langle a_{1}\right\rangle$ or the modulation depth [24], [25] $D=1-\left\langle a_{0}\right\rangle /\left\langle a_{1}\right\rangle$. For example, when $\left\langle a_{1}\right\rangle /\left\langle a_{0}\right\rangle=50, E=1: 50$ and $D=98$ percent.
}

electron counting distributions for Fig. 7. In calculating the curves, the optimal decision regions (specified by the solutions to (26)) were redetermined for each value of $\left\langle a_{1}\right\rangle /\left\langle a_{0}\right\rangle .{ }^{10}$ Error-probability curves are also presented for the ideal Poisson photoelectron counter without multiplication noise (solid curve labeled Poisson). This corresponds to the shot-noise-limited receiver. Results are displayed for the three-stage SAPD in Fig. 7 and for the ten-stage SAPD in Fig. 8. The electron ionization probability $P$ used to compute each SAPD curve is indicated beside it. Similarly, the value of $y$ used to compute each CAPD curve is indicated. Every value of $y$ is accompanied by a value of $P$ (in parentheses) that provides equivalent gain in accordance with (25).

Figs. 7 and 8 illustrate that, for multiplication-noiselimited operation, the performance associated with the single-carrier SAPD improves as $P$ increases. This result is true for both values of $m$, as expected. Indeed, the error probability approaches that of the ideal Poisson photon counter as $P$ moves close to unity. In this limit the superlattice device behaves as a noiseless electron multiplier so that shot-noise-limited operation is attained. For $P=$ 0.95 , little noise is introduced by the device. A comparison of the dotted curves in Figs. 7 and 8 shows that, for fixed $P$, performance is degraded as $m$ increases. The degradation is negligible, however, for $P=0.95$. This is important since high values of $m$ provide large gain.

The performance of the SCISCM SAPD receiver is always superior to that of the SCISCM CAPD receiver in the multiplication-noise-limited regime. Indeed, performance becomes identical in the limit $P \rightarrow 0$ (with $(1+$ $P)^{m}$ finite) [2]. The performance of the CAPD system is seen to degrade as $y$ increases. This reflects the behavior of the Poisson-driven Yule-Furry process; it becomes progressively noisier as the branching parameter (and therefore the gain) increase. The superlattice device, on the other hand, becomes progressively less noisy as $P$ approaches unity. The improvement provided by the superlattice device therefore widens as $P$ increases (for fixed $m)$. There is also an increasing advantage for the SAPD as $m$ increases (for fixed $P$ ) and as $\left\langle a_{1}\right\rangle /\left\langle a_{0}\right\rangle$ increases (for fixed $m$ and $P$ ). Although probability of error curves for a multiplication-noise-limited OOK system incorporating a PMT are available [51], they are not included here because our principal emphasis is on a comparison of the CAPD and SAPD receivers.

Another way of presenting the performance results reported above is in terms of receiver sensitivity. The most

\footnotetext{
${ }^{10}$ The Poisson-driven Yule-Furry counting distribution is not always monotonically decreasing (this depends on the parameters $\langle a\rangle$ and $y$ ), although the three specific examples illustrated in Fig. 2 do have this property. When the logarithm of the noise counting distribution does not contain a point of inflection, as in Fig. 2, comparison with a unique threshold comprises optimal processing [50]. The SAPD noise log-probability distributions (Fig. 4), on the other hand, display points of inflection so that the unique-threshold theorem [50] cannot be applied. Nevertheless, for all of the SAPD signal and noise distributions calculated in this paper, it was empirically determined that comparison with a single threshold was indeed optimal.
} 


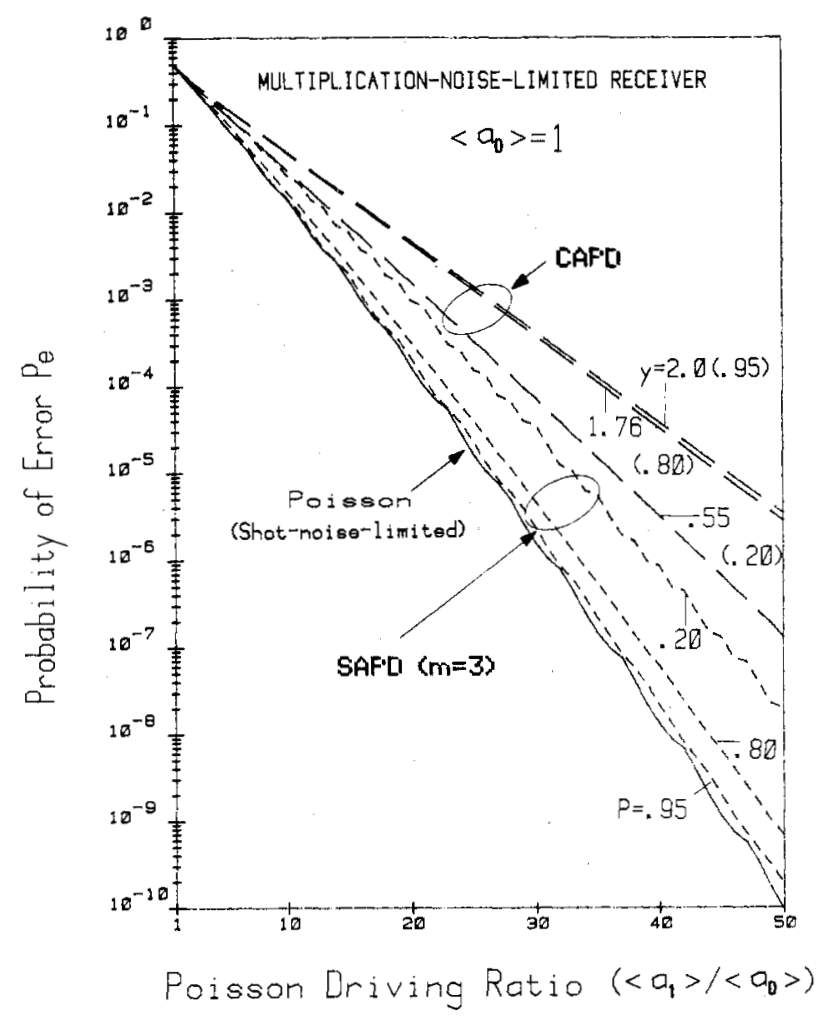

Fig. 7. Error probability $P_{e}$ versus Poisson driving ratio $\left\langle a_{1}\right\rangle /\left\langle a_{0}\right\rangle$ in the multiplication-noise-limited regime. The mean number of injected noise photoelectrons per pulse $\left\langle a_{0}\right\rangle=1.0$ for all cases. Curves are presented for the ideal Poisson photoelectron counter without multiplication noise (solid curve labeled Poisson), for the CAPD (dashed curves), and for the three-stage SAPD (dotted curves). The electron ionization probability $P$ used to compute each SAPD curve is indicated beside it. Similarly, the value of $y$ used to compute each CAPD curve is indicated. Every value of $y$ is accompanied by a value of $P$ (in parentheses) that provides equivalent mean gain. The performance of the system incorporating the threestage SAPD is seen to be nearly ideal for $P=0.95$.

commonly used sensitivity measure is the averge number of photons per bit $\langle N\rangle$ required to achieve a given bit error rate (BER $\equiv P_{e}$ ), conventionally $10^{-9}$. When $\left\langle a_{0}\right\rangle$ $=1$, the average number of detected photons/pulse $\left\langle a_{1}\right\rangle$ required to achieve $P_{e}=10^{-9}$ may be estimated from extended versions of Figs. 7 and 8. For maximum-likelihood detection with binary OOK, $\langle N\rangle=\left\langle a_{1}\right\rangle / 2 \eta$. Receiver sensitivities for SAPD and CAPD optical receivers are presented in Table I for various values of the average gain $\langle M\rangle$ when $\left\langle a_{0}\right\rangle=1$ and $\eta=1$. For the shot-noiselimited (Poisson) receiver, $\langle N\rangle=22.5$ (this result is attained by the SAPD receiver with $P=1$ ). Also shown are values for a normalized sensitivity measure $\langle N\rangle / F_{e}$ similar to that used by Webb et al. [24]. $\langle N\rangle / F_{e}$ is seen to be more or less constant over the range of parameters presented, lying between 22 and 23. However, its value is strongly dependent on $\left\langle a_{0}\right\rangle$.

In the absence of background radiation and dark noise $\left(\left\langle a_{0}\right\rangle=0\right)$, and when there is no thermal noise present, $q\left(n \mid H_{0}\right)=\delta(n)$. False alarms are not possible in this case. The sensitivity of the unity-quantum-efficiency binary OOK receiver is then limited only by the quantum fluctuations of the signal light. It is immaterial whether mul-

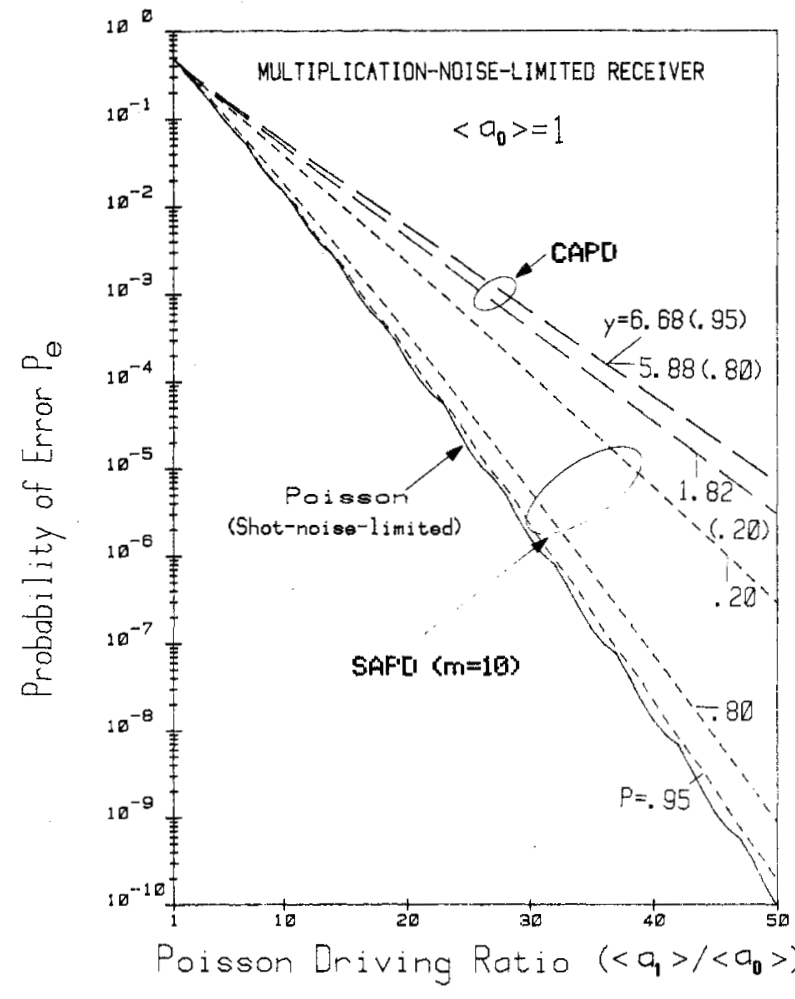

Fig. 8. Error probability $P_{e}$ versus Poisson driving ratio $\left\langle a_{1}\right\rangle /\left\langle a_{0}\right\rangle$ in the multiplication-noise-limited regime. The mean number of injected noise photoelectrons per pulse $\left\langle a_{0}\right\rangle=1.0$ for all cases. Curves are presented for the ideal Poisson photoelectron counter, for the CAPD (dashed curves), and for the ten-stage SAPD (dotted curves) with the same mean gain as the CAPD. System performance with the ten-stage SAPD is seen to be just about as good as that for the three-stage SAPD for $P=0.95$ (see Fig. 7),

TABLE I

Receiver Sensttivity $\langle N\rangle$ (Average Number of Photons Per Bit) REQUIRED TO ACHIEVE $P_{e}=10^{-9}$ WHEN $\left\langle a_{0}\right\rangle=1$ AND $\eta=1$ (The binary OOK optical receiver is assumed to be multiplication-noise limited. Values for the figure of merit $\langle N\rangle / F_{e}$ are also shown.)

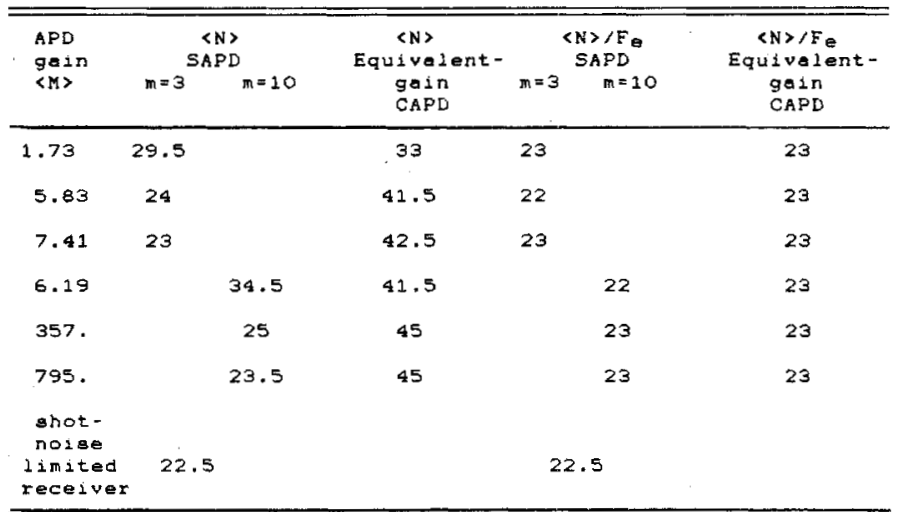

tiplication noise is present: a miss can only be achieved by the absence of photons at the input and there are no false alarms. From (26a), the only integer element lying in $Z_{0}$ is zero. Using (27) and (29), together with (16) or (21), the probability of error becomes ${ }^{11} P_{e}=\frac{1}{2} P_{M}=$

\footnotetext{
${ }^{11}$ The prefactor $\frac{1}{2}$ is sometimes (improperly) omitted, resulting in a direct-detection quantum limit quoted as 10.5 photons/bit instead of the correct value of 10 photons/bit.
} 
$\frac{1}{2} q\left(0 \mid H_{1}\right)=\frac{1}{2} \exp \left(-\left\langle a_{1}\right\rangle\right)=\frac{1}{2} \exp (-2\langle N\rangle)$. Setting $P_{e}=10^{-9}$, in accord with convention, gives rise to the so-called direct-detection quantum limit of 10 photons/bit for OOK. In the presence of noise specified by $\left\langle a_{0}\right\rangle=1$, on the other hand, the best that can be achieved is $\langle N\rangle$ $=22.5$ photons/bit, as is apparent from Table I. The presence of noise increases $\langle N\rangle$. From an experimental point of view, the state-of-the-art sensitivity for a 0.85 $\mu \mathrm{m}$ Si APD receiver, operating at a speed of several hundred megabits per second, lies roughly at 300 photons/bit. In the 1.3- to $1.6-\mu \mathrm{m}$ region, on the other hard, it is about 1000 photons/bit [52].

In the next section, we consider the deleterious effects of thermal noise on receiver performance.

VII. COMPARISON OF CAPD AND SAPD OPTICAL Receiver Performance in the Presence of Thermal NOISE

The effects of thermal (Johnson) noise are incorporated into the receiver model by means of an additive independent zero-mean Gaussian random variable $j$ representing the thermal-noise current fluctuations [1], [27], [33], [38], [53]-[56]. Its probability density is

$$
p(j)=\left(2 \pi \sigma^{2}\right)^{-1 / 2} \exp \left(-j^{2} / 2 \sigma^{2}\right)
$$

with mean

$$
\langle j\rangle=0
$$

and variance (thermal noise power)

$$
\operatorname{Var}(j)=\sigma^{2}=4 k \theta B / R_{L} .
$$

The quantity $R_{L}$ represents the effective load resistance ${ }^{12}$ at temperature $\theta, k$ is Boltzmann's constant, and $B$ is the effective electrical bandwidth of the system. For ideal photon counting

$$
B=1 / 2 T
$$

where $T$ is the counting (observation) time [23] so that (32) can be rewritten as

$$
\sigma^{2}=2 k \theta / R_{L} T
$$

The probability density of the total current (multiplied shot noise plus thermal noise) under hypotheses $H_{0}$ ard $H_{1}$ is then described by the convolution

$$
\begin{aligned}
q\left(i \mid H_{0,1}\right)= & \frac{1}{\left(2 \pi \sigma^{2}\right)^{1 / 2}} \\
& \cdot \sum_{n=0}^{\infty} q\left(n \mid H_{0,1}\right) \exp \left[-\left(i-\frac{e n}{T}\right)^{2} / 2 \sigma^{2}\right]
\end{aligned}
$$

where $q\left(n \mid H_{0,1}\right)=q_{y}\left(n \mid H_{0,1}\right)$ or $q_{m}\left(n \mid H_{0,1}\right)$, depending on whether the detector is a CAPD or an SAPD. The fac-

\footnotetext{
${ }^{12}$ The value of $R_{L}$ depends significantly on the nature of the preamplifier, e.g., whether it is a high-impedance integrating front end or a transimped-. ance amplifier [1], [53], [54], [57].
}

tor $(e / T)$ converts count number to current. The variance $\sigma^{2}$, which arises from the Gaussian thermal noise, is the same independent of which hypothesis is true. Again, the mean numbers of Poisson injected photoelectrons per pulse are taken to be $\left\langle a_{0}\right\rangle$ and $\left\langle a_{1}\right\rangle$ under hypotheses $H_{0}$ and $H_{1}$, respectively.

Using (15a), (19), and (31), the mean values of the total current are then

$$
\begin{aligned}
& \left\langle i \mid H_{0,1}\right\rangle=(e / T)\left\langle a_{0,1}\right\rangle \exp (y) \\
& \left\langle i \mid H_{0,1}\right\rangle=(e / T)\left\langle a_{0,1}\right\rangle(1+P)^{m}
\end{aligned}
$$

for the CAPD and SAPD, respectively. The variances of the total current for the CAPD and SAPD are obtained by multiplying $(15 \mathrm{~b})$ and $(20)$ by $\left(e^{2} / T^{2}\right)$ (to convert count number variances to current variances) and then adding the thermal noise current variance (34). The results are

$$
\operatorname{Var}\left(i \mid H_{0,1}\right)=\left(e^{2} / T^{2}\right) \operatorname{Var}\left(n_{y} \mid\left\langle a_{0,1}\right\rangle\right)+2 k \theta / R_{L} T
$$

$$
\operatorname{Var}\left(i \mid H_{0,1}\right)=\left(e^{2} / T^{2}\right) \operatorname{Var}\left(n_{m} \mid\left\langle a_{0,1}\right\rangle\right)+2 k \theta / R_{L} T
$$

for the CAPD and SAPD, respectively. It is convenient to define $R$ as the ratio of the thermal-noise current variance to the multiplication-noise current variance under $H_{0}$, i.e.

$$
\begin{aligned}
& R_{y}=2 k \theta T / e^{2} R_{L} \operatorname{Var}\left(n_{y} \mid\left\langle a_{0}\right\rangle\right) \\
& R_{m}=2 k \theta T / e^{2} R_{L} \operatorname{Var}\left(n_{m} \mid\left\langle a_{0}\right\rangle\right)
\end{aligned}
$$

for the CAPD and SAPD, respectively.

To implement maximum-likelihood detection, in analogy with (26), the observation space $Z$ is divided into two parts, $Z_{0}$ and $Z_{1}$, such that

$$
\begin{aligned}
& Z_{0}=\left\{i \mid q\left(i \mid H_{0}\right) \geq q\left(i \mid H_{1}\right)\right\} \\
& Z_{1}=\left\{i \mid q\left(i \mid H_{0}\right)<q\left(i \mid H_{1}\right)\right\} .
\end{aligned}
$$

When an observation falls in $Z_{0}$, we say $H_{0}$; when it falls in $Z_{1}$, we say $H_{1}$. The error probability $P_{e}$ is given by (27), in terms of the false-alarm and miss probabilities. In analogy with (28) and (29) these are

$$
P_{F}=\int_{Z_{1}} q\left(i \mid H_{0}\right) d i
$$

and

$$
P_{M}=\int_{Z_{0}} q\left(i \mid H_{1}\right) d i
$$

respectively. Using (35), these probabilities can be written explicitly as

$$
P_{F}=\frac{1}{2}-\frac{1}{2} \sum_{n=0}^{\infty} q\left(n \mid H_{0}\right) \operatorname{erf}\left[(\Phi-e n / T) /\left(2 \sigma^{2}\right)^{1 / 2}\right]
$$




$$
P_{M}=\frac{1}{2}+\frac{1}{2} \sum_{n=0}^{\infty} q\left(n \mid H_{1}\right) \operatorname{erf}\left[(\Phi-e n / T) /\left(2 \sigma^{2}\right)^{1 / 2}\right]
$$

where

$$
\Phi=\left\{\Phi \mid q\left(\Phi \mid H_{0}\right)=q\left(\Phi \mid H_{1}\right)\right\}
$$

and

$$
\operatorname{erf}(x)=\frac{2}{\pi^{1 / 2}} \int_{0}^{x} \exp \left(-t^{2}\right) d t
$$

The bit error rate $P_{e}$ is determined by (27), using (42)(45). In Fig. 9 we plot $P_{e}$ versus the Poisson driving (signal-to-noise) ratio ra $^{9}\left\langle a_{1}\right\rangle /\left\langle a_{0}\right\rangle$ for the SAPD $(m=3, P$ $=0.95)$ and the CAPD of the same mean $(y=2.0)$. As previously, the mean number of injected photoelectrons per pulse, under hypothesis $H_{0}$, is chosen to be exactly $\left\langle a_{0}\right\rangle=1.0$ for all cases. In calculating the curves, the optimal decision regions (specified by the solutions to (39)) are redetermined for each value of $\left\langle a_{1}\right\rangle /\left\langle a_{0}\right\rangle .{ }^{10}$ The parameter $R$ represents the thermal noise power, in terms of the ratio specified in (38). System performance is determined by calculating the value of $R$ for the components in the system and then consulting the appropriate curve for $R_{m}$ or $R_{y}$ in Fig. 9.

The $R=0$ curves represent the absence of thermal noise; they are therefore the same as those shown in Fig. 7. As expected, the probability of error increases as the thermal-noise ratio increases. For $R=500$ (which is easily achievable for the gain values considered here), the deterioration in performance is enormous. In the extreme case of thermal-noise-limited operation $(R \rightarrow \infty)$, the SAPD and CAPD curves will coincide since the multiplication-noise contribution is then negligible. The performance of the SAPD receiver is superior to that of the CAPD receiver for $R_{m}=R_{y}$. This is expected since the current variance of the SAPD always lies below that of the equivalent-gain CAPD. ${ }^{13}$ Again, the probability of error curve for the ideal shot-noise-limited Poisson photoelectron counter, with neither multiplication nor thermal noise, is displayed in Fig. 9 as the solid curve labeled Poisson.

There have been numerous studies of the performance of optical receivers incorporating double-carrier CAPD's in the presence of thermal noise. Some of these have involved exact numerical calculations or Monte Carlo simulations [58], [59]. More often, because of the complexity of the calculations, researchers have resorted to approximate solutions. These have included the use of direct mathematical approximations [24], Chernoff bounding [58], and the use of the Gaussian approximation [58].

\footnotetext{
${ }^{13}$ For convenience of presentation, the effect of thermal noise has been incorporated into the ratio of its current variance to the multiplication-noise current variance. If the thernal-noise variance were instead assumed to be a fixed quantity determined by the characteristics of the preamplifier, the appropriate comparison would be between different values of $R_{m}$ and $R_{y}$ and the performance advantage of the SAPD would then not be as great.
}

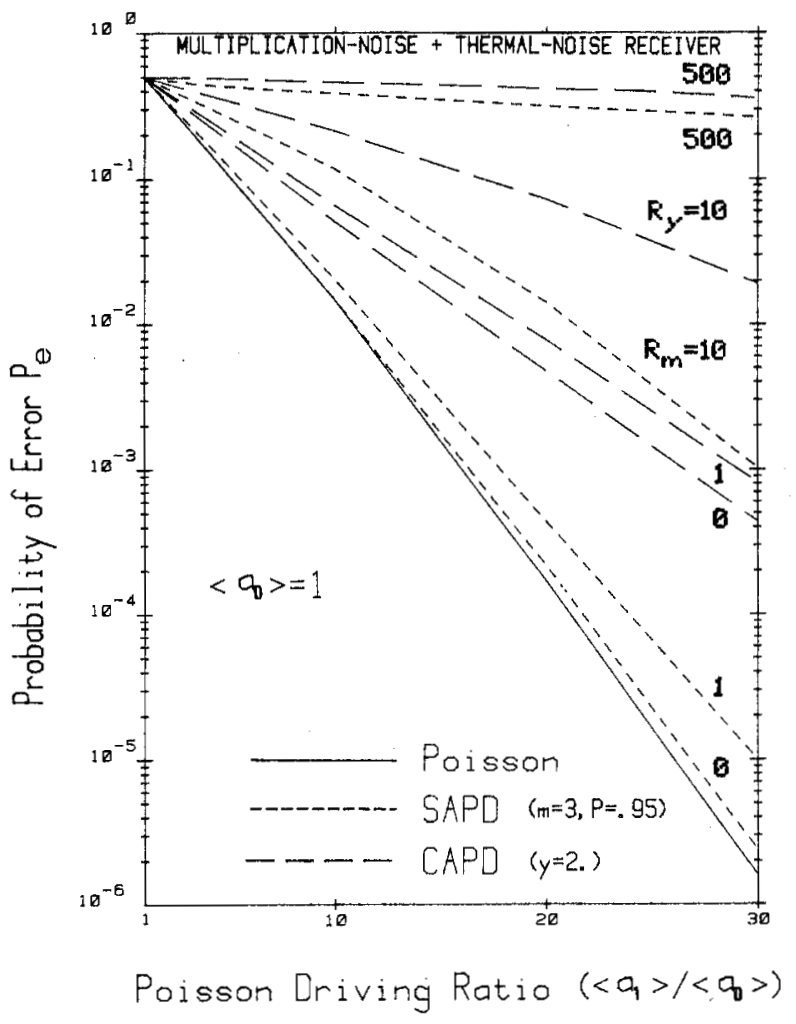

Fig. 9. Error probability $P_{e}$ versus Poisson driving ratio $\left\langle a_{1}\right\rangle\left\langle a_{0}\right\rangle$ for the SAPD ( $m=3, P=0.95)$ and the CAPD of the same mean $(y=2.0)$ in the presence of thermal noise. The mean number of injected noise photoelectrons per pulse $\left\langle a_{0}\right\rangle=1.0$ for all cases. The parameter $R$ is the ratio of the thermal-noise current variance to the multiplication-noise current variance under $H_{0}$. Performance is determined by calculating the value of $R$ for the system components under consideration and consulting the appropriate curve for $R_{m}$ or $R_{y}$. The $R=0$ curves represent the absence of thermal noise and correspond to those shown in Fig. 7. Increasing thermal noise causes a deterioration in performance.

This latter approach has the advantage of ease of computation [53], [54], but can suffer from substantial inaccuracies [24], [58], [60]. Other methods have included use of the Gaussian quadrature rule [61], the GramCharlier series [62], and the saddlepoint approximation [63].

\section{Discussion AND CONClusion}

Exact gain distributions and electron counting distributions (under Poisson carrier injection) have been calculated and displayed for single-carrier CAPD's, singlecarrier SAPD's, and a single-stage PMT. The shapes of these distributions have been shown to differ in characteristic ways for the three devices. In particular, the SAPD counting distribution can become quite scalloped for even moderate values of $P$ and $m$. This could be a useful feature in providing good pulse-height resolution. The electron counting distributions were used to numerically calculate the probability of error achievable by a simple digital optical communication system. A performance comparison was carried out for a maximum-likelihood integrate-and-dump OOK optical receiver incorporating 
the lowest-possible noise (single-carrier) CAPD's and SAPD's, under conditions of identical quantum efficiencies and gains.

System performance with the superlattice device is always superior to that attainable with the conventional APD for all values of the gain. The advantage, which grows with increasing $P, m$, and Poisson driving ratio $\left\langle a_{1}\right\rangle /\left\langle a_{0}\right\rangle$, can attain several orders of magnitude, even though the excess noise factors for the two devices differs at most by a factor of two. The single-carrier SAPD with high impact-ionization probability behaves like a shotnoise-limited detector with the same quantum efficiency, irrespective of the number of stages of the device $m$. The performance degradation caused by thermal noise is, if anything, less severe for the SAPD.

Our comparison has been restricted to the SCISCM CAPD and SAPD since only single-carrier counting cistributions are available for the SAPD. The advantages of the SAPD optical receiver presented here follow from its discrete-branching mechanism, which provides decreased noise. The presence of double-carrier multiplication would degrade both CAPD and SAPD receiver performance substantially [2].

From an experimental point of view, multiquantum-well SAPD's have been fabricated and are being tested [3][10] and the first attempts are now under way to construct staircase SAPD devices using molecular-beam epitcxy [22]. From a theoretical point of view, there are a number of extensions of the theory that are possible. The effects of additive non-multiplying and multiplying dark noise could be incorporated into the performance calculations. Account could be taken of double-carrier multiplication. Other signaling formats and receiver structures could be investigated. Intersymbol interference could be incluced [64]. The consequences of non-Poisson photon excitation could be calculated. The effects of nonuniform APD gain [65] could also be considered.

Finally, we point out that it may be of interest to consider the possibility of constructing a high-speed SAl?D photocounting receiver. In receivers of this type, charge pulses arising from individual injected electrons are sufficiently well separated in time that they can be individually counted. The PMT has long been used in this mode [23], and more recently so have CAPD's [3], [37], [66][72]. Operating the SAPD as a photon counter may present difficulties, however, since $10^{4}$ electrons/photon are generally required to overcome pre-amplifier Johnson noise [4]. The gain of the single-carrier SAPD is limied to $2^{m}$, so that a high- $P$ single-carrier device with some 15 stages of gain would be required [4].

\section{ACKNOWLEDGMENT}

It is a pleasure to thank R. J. McIntyre, F. Capasiso, and $\mathrm{K}$. Brennan for useful discussions.

\section{REFERENCES}

[1] S. D. Personick, Fiber Optics. New York: Plenum, 1985.

[2] M. C. Teich, K. Matsuo, and B. E. A. Saleh, "Excess noise factors for conventional and superlattice avalanche photodiodes and photomultiplier tubes," IEEE J. Quant. Electron., vol, QE-22, pp. 1184$1193,1986$.

[3] P. P. Webb and R. J. McIntyre, "Recent developments in silicon avalanche photodiodes," RCA Eng., vol. 27, pp. 96-102, 1982.

[4] R. J. McIntyre, RCA Electro-Optics Photodetectors, Ste. Anne de Bellevue, P.Q., Canada, personal communication.

[5] G. E. Stillman, V. M. Robbins, and N. Tabatabaie, "III-V compound semiconductor devices: Optical detectors," IEEE Trans. Electron Devices, vol. ED-31, pp. 1643-1655, 19.84.

[6] F. Capasso, "Physics of avalanche photodiodes," in Semiconductors and Semimetals, R. K. Willardson and A. C. Beer, Series Eds., vol. 22, part D, Lightwave Communications Technology, W. T. Tsang, Ed. New York: Academic, 1985, pp. 1-172.

[7] R. Chin, N. Holonyak, G. E. Stillman, J. Y. Tang, and K. Hess, "Impact ionization in multilayered heterojunction structures," Electron. Lett., vol. 16, pp. 467-469, 1980.

[8] F. Capasso, W. T. Tsang, A. L. Hutchinson, and G. F. Williams, "Enhancement of electron impact ionization in a superlattice: A new avalanche photodiode with a large ionization rate ratio," Appl. Phys. Lett., vol, 40, pp. 38-40, 1982.

[9] K. Mohammed, F. Capasso, J. Allam, A. Y. Cho, and A. L. Hutchinson, "New high-speed long-wavelength $\mathrm{Al}_{0.48} \mathrm{In}_{0.52} \mathrm{As}$ / $\mathrm{Ga}_{0.47} \mathrm{In}_{0.53}$ As multiquantum well avalanche photodiodes," Appl. Phys. Lett., vol. 47, pp. 597-599, 1985.

[10] F.-Y. Juang, U. Das, Y. Nashimoto, and P. K. Bhattacharya, "Electron and hole impact ionization coefficients in $\mathrm{GaAs}-\mathrm{Al}_{x} \mathrm{Ga}_{1-x} \mathrm{As}$ superlattices," Appl. Phys. Lett., vol. 47, pp. 972-974, 1985.

[11] K. Brennan, "Theory of electron and hole impact ionization in quantum well and staircase superlattice avalanche photodiode structures," IEEE Trans. Electron Devices, vol. ED-32, pp. 2197-2205, 1985.

[12] — , "Theoretical study of multiquantum well avalanche photodiodes made from the GaInAs/AlInAs material system, " IEEE Trans. Electron Devices, this issue, pp. 1502-1510.

[13] G. F. Williams, F. Capasso, and W. T. Tsang, "The graded bandgap multilayer avalanche photodiode: A new low-noise detector," IEEE Electron Device Lett., vol. EDL-3, pp. 71-73, 1982.

[14] F. Capasso, W. T. Tsang, and G. F. Williams, "Staircase solid-state photomultipliers and avalanche photodiodes with enhanced ionization rates ratio," IEEE Trans. Electron Devices, vol. ED-30, pp. 381 $390,1983$.

[15] F. Capasso, "Band-gap engineering via graded gap, superlattice, and periodic doping structures: Applications to novel photodetectors and other devices," J. Vac. Sci. Tech. B, ser. 2, vol. 1, pp. 457-461, 1983.

[16] K. Matsuo, M. C. Teich, and B. E. A. Saleh, "Noise properties and time response of the staircase avalanche photodiode," IEEE Trans. Electron Devices, vol. ED-32, pp. 2615-2623, 1985; also in J. Lightwave Technol, , vol. LT-3, pp. 1223-1231, 1985.

[17] H. Blauvelt, S. Margalit, and A. Yariv, "Single-carrier-type dominated impact ionisation in multilayer structures, ' Electron. Lett., vol, 18, pp. 375-376, 1982.

[18] K. Brennan, "Theory of the GaInAs/AlInAs doped-quantum-well APD: A new low-noise solid-state photodetector for lightwave communications systems," to be published.

[19] J. S. Smith, L. C. Chiu, S. Margalit, A. Yariv, and A. Y. Cho "A new infrared detector using electron emission from multiple quantum wells," J. Vac. Sci. Technol. B, vol. 1, pp. 376-378, 1983.

[20] S. L. Chuang and K. Hess, "Impact ionization across the conductionband-edge discontinuity of quantum-well heterostructures," J. Appl. Phys., vol. 59, pp. 2885-2894, 1986.

[21] F. Capasso, J. Allam, A. Y. Cho, K. Mohammed, R. J. Malik, A. L. Hutchinson, and D. Sivco, "New avalanche multiplication phenomenon in quantum well superlattices: Evidence of impact ionization across the band-edge discontinuity," Appl. Phys. Lett., vol. 48, pp. 1294-1296, 1986.

[22] F. Capasso, AT\&T Bell Laboratories, Murray Hill, NJ, private communication.

[23] R. W. Engstrom, RCA Photomultiplier Handbook (PMT-62). Lancaster, PA, RCA Electro Optics and Devices, 1980.

[24] P. P. Webb, R. J. McIntyre, and J. Conradi, "Properties of avalanche photodiodes," RCA Rev, , vol, 35, pp. 234-278, 1974.

[25] G. E. Stillman and C. M. Wolfe, "Avalanche photodiodes," in Semiconductors and Semimetals, vol. 12, Infrared Detectors II, R. K. Willardson and A. C. Beer, Eds. New York: Academic, 1977, pp. 291-394.

[26] H. L. van Trees, Detection, Estimation, and Modulation Theory, Part I. New York: Wiley, 1968. 
[27] W. K. Pratt, Laser Communication Systems. New York: Wiley, 1969.

[28] L. Mandel, "Image fluctuations in cascade intensifiers, ' Brit. J. Appl. Phys., vol. 10, pp. 233-234, 1959.

[29] R. E. Burgess, "Some topics in the fluctuations of photo-processes in solids," J. Phys. Chem. Solids, vol. 22, pp. 371-377, 1961.

[30] K. M. van Vliet and L. M. Rucker, "Noise associated with reduction, multiplication and branching processes," Physica, vol. 95A, pp. 117140,1979

[31] M. C. Teich and B. E. A. Saleh, "Effects of random deletion and additive noise on bunched and antibunched photon-counting statistics," Opt. Lett., vol. 7, pp. 365-367, 1982.

[32] E. Parzen, Stochastic Processes. San Francisco: Holden-Day, 1962.

[33] S. D. Personick, "New results on avalanche multiplication statistics with application to optical detection," Bell Syst. Tech. J., vol. 50, pp. $167-189,1971$.

[34] K. Matsuo, M. C. Teich, and B. E. A. Saleh, "Poisson branching point processes," J. Math. Phys., vol. 25, pp. 2174-2185, 1984.

[35] G. U. Yule, "A mathematical theory of evolution, based on the conclusions of Dr. J. C. Willis, F. R. S.," Phil. Trans. Royal Soc. London, vol. B213, pp. 21-87, 1924.

[36] W. Furry, "On fluctuation phenomena in the passage of high energy electrons through lead," Phys. Rev., vol. 52, pp. 569-581, 1937.

[37] R. J. McIntyre, "The distribution of gains in uniformly multiplying avalanche photodiodes: Theory,'"IEEE Trans. Electron Devices, vol. ED-19, pp. 703-713, 1972.

[38] S. D. Personick, "Statistics of a general class of avalanche detectors with applications to optical communication," Bell Syst. Tech. J., vol. 50, pp. 3075-3095, 1971.

[39] J. Conradi, "The distribution of gains in uniformly multiplying avalanche photodiodes: Experimental," IEEE Trans. Electron Devices, vol. ED-19, pp. 713-718, 1972.

[40] S. K. Srinivasan, Stochastic Theory and Cascade Processes. New York: Elsevier, 1969.

[41] C. W. Helstrom, "Computation of output electron distributions in avalanche photodiodes," IEEE Trans. Electron Devices, vol. ED-31, pp. 955-958, 1984.

[42] F. Capasso, "The channeling avalanche photodiode: A novel ultralow-noise interdigitated p-n junction detector," IEEE Trans, Electron Devices, vol. ED-29, pp. 1388-1395, 1982.

[43] K. Brennan, "Theory of the channeling avalanche photodiode," IEEE Trans. Electron Devices, vol. ED-32, pp. 2467-2478, 1985.

[44] M. C. Teich, "Role of the doubly stochastic Neyman type-A and Thomas counting distributions in photon detection," Appl. Opt., vol. 20, pp. $2457-2467,1981$.

[45] K. Matsuo, B. E. A. Saleh, and M. C. Teich, "Cascaded Poisson processes," J. Math. Phys., vol. 23, pp. 2353-2364, 1982.

[46] L. Janossy, Zh. Eksperim. Teor. Fiz., vol. 28, p. 679, 1955.

[47] _ , "Statistical problems of an electron multiplier," Sov. Phys. JETP, vol. 1, pp. 520-531, 1955.

[48] F. J. Lombard and F. Martin, "Statistics of electron multiplication," Rev. Sci. Instr., vol. 32, pp. 200-201, 1961.

[49] J. Neyman, 'On a new class of 'contagious' distributions, applicable in entomology and bacteriology," Ann. Math. Stat., vol. 10, pp. 35$57,1939$.

[50] P. R. Prucnal and M. C. Teich, "Single-threshold detection of a random signal in noise with multiple independent observations. 1: Discrete case with application to optical communications," Appl. Opt., vol. 17 , pp. 3576-3583, 1978.

[51] G. Lachs, "The statistics for the detection of light by nonideal photomultipliers,"' IEEE J. Quant. Electron., vol. QE-10, pp. 590-596, 1974.

[52] P. S. Henry, "Lightwave primer," IEEE J. Quant. Electron., vol. QE-21, pp. 1862-1879, 1985.

[53] S: D. Personick, "Receiver design for optical systems,"' Proc. IEEE, vol. 65 , pp. $1670-1678,1977$

[54] S. D. Personick, "Receiver design," in Optical Fiber Telecommunications, S. E. Miller and A. G. Chynoweth, Eds. New York: Academic, 1979, pp. 627-651

[55] R. G. Smith and S. D. Personick, "Receiver design for optical fiber communication systems," in Semiconductor Devices for Optical Communication, 2nd ed., H. Kressel, Ed. New York: Springer-Verlag, 1982 .

[56] J. M. Senior, Optical Fiber Communications, Englewood Cliffs, NJ; Prentice-Hall, 1985

[57] J. L. Hullet and T. V. Muoi, "A feedback receiver amplifier for optical transmission systems,"' IEEE Trans. Commun., vol. COM-24, pp. 1180-1185, 1976 .
[58] S. D. Personick, P. Balaban, J. H. Bobsin, and P. R. Kumar, “A detailed comparison of four approaches to the calculation of the sensitivity of optical fiber system receivers," IEEE Trans. Commun., vol. COM-25, pp. 541-548, 1977.

[59] N. Sorensen and R. Gagliardi, "Performance of optical receivers with avalanche photodetection," IEEE Trans. Commun., vol. COM-27, pp. 1315-1321, 1979.

[60] B. L. Kasper, J. C. Campbell, and A. G. Dentai, "Measurements of the statistics of excess noise in separate absorption, grading and multiplication (SAGM) avalanche photodiodes,' Electron. Lett., vol. 20, pp. 796-798, 1984 .

[61] W. Hauk, F. Bross, and M. Ottka, "The calculation of error rates for optical fiber systems," IEEE Trans. Commun., vol. COM-26, pp. 1119-1126, 1978.

[62] M. Mansuripur, J. W. Goodman, E. G. Rawson, and R. E. Norton, "Fiber optics receiver error rate prediction using the Gram-Charlier series," IEEE Trans. Commun., vol. COM-28, pp. 402-407, 1980.

[63] D. Källgren, "On error probability in a fiber optic channel with an approximate avalanche gain mechanism," IEEE Conf. Communications Conf. Rec., vol. 2, pp. 547-551, 1985.

[64] J. E. Mazo and J. Salz, "On optical data communication via direct detection of light pulses,' Bell Syst. Tech. J., vol. 55, pp. 347-369, 1976.

[65] Y. K. Jhee, J. C. Campbell, W. S. Holden, A. G. Dentai, and J. K. Plourde, "The effect of nonuniform gain on the multiplication noise of InP/InGaAsP/InGaAs avalanche photodiodes,"' IEEE J. Quantum Electron., vol. QE-21, pp. 1858-1861, 1985.

[66] D. O. Cummings, "Simulation of thermal noise at optical frequencies and use of an avalanche photodiode for high speed photon counting," Ph.D. dissertation Pennsylvania State Univ., State College, 1972.

[67] W. Fichtner and W. Häcker, "Time resolution of Ge avalanche photodiodes operating as photon counters in delayed coincidence," Rev. Sci. Instrum., vol. 47, pp. 374-377, 1976.

[68] P. A. Ekstrom, "Triggered avalanche detection of optical photons ", J. Appl. Phys., vol. 52, pp. 6974-6979, 1981.

[69] B. F. Levine and C. G. Bethea, "Single photon detection at $1.3 \mu \mathrm{m}$ using a gated avalanche photodiode," Appl. Phys. Lett., vol. 44, pp. 553-555, 1984.

[70] B. F. Levine and C. G. Bethea, "Error rate measurement for single photon detection at $1.3 \mu \mathrm{m}, "$ Appl. Phys. Lett., vol. 44, pp. $649-$ $650,1984$.

[71] B. F. Levine and C. G. Bethea, "Detection of single $1.3 \mu \mathrm{m}$ photons at $45 \mathrm{Mbit} / \mathrm{s}$,' Electron. Lett., vol. 20, pp. 269-271, 1984.

[72] K. Kikuchi, T. Okoshi, and A. Hirose, "Achievement of shot-noiselimited sensitivity and $50 \mathrm{~dB}$ dynamic range by photon-counting receiver using Si avalanche photodiode, "Electron. Lett., vol. 21, pp. $801-802,1985$.

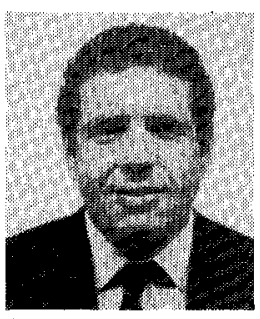

Malvin C. Teich (S'62-M'66-SM'72) was born in New York, NY. He received the S.B. degree in physics from the Massachusetts Institute of Technology, Cambridge, in 1961 , the M.S. degree in electrical engineering from Stanford University, Stanford, CA, in 1962, and the Ph.D. degree in quantum electronics from Cornell University, Ithaca, NY, in 1966.

In 1966 he joined the MIT Lincoln Laboratory, Lexington, MA, where he was engaged in work on coherent infrared detection. In 1967 , he became a member of the faculty in the Department of Electrical Engineering, Columbia University, New York, NY, where he is now teaching and pursuing his research interests in the areas of optical and infrared detection, quantum optics, lightwave communications, and sensory perception. $\mathrm{He}$ served as Chairman of the Department from 1978 to 1980 . He is also a member of the faculty in the Department of Applied Physics and Nuclear Engineering, and a member of the Columbia Radiation Laboratory, the Center for Telecommunications Research, and the Columbia Bioengineering Institute. He has authored or coauthored some 100 technical publications and holds one patent.

Dr. Teich is a member of Sigma Xi, the American Physical Society, the Acoustical Society of America, the Society for Neuroscience, the American Association for the Advancement of Science, and the New York Academy of Sciences. He served as a member of the Editorial Advisory Panel for Optics Letters from 1977 to 1979 . In 1969 he was the recipient of the 
IEEE Browder J. Thompson Memorial Prize for his paper " Infrared Heterodyne Detection"' and in 1981 he received the Citation Classic Award of Current Contents for this work. He was appointed a Fellow of the John Simon Guggenheim Memorial Foundation in 1973 and was elected a Fellow of the Optical Society of America in 1983.

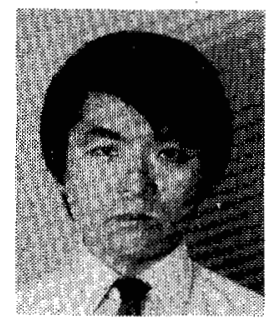

Kuniaki Matsuo (M'85) was born in Hiroshima, Japan. He received the B.S. degree in electrical engineering from Tokyo Electrical Engineering College, Tokyo, in 1972 and the M.S. and Ph.D. degrees in electrical engineering from Colu nbia University, New York, NY, in 1978 and 1984, respectively.

From 1984 to 1986 he was a Postdoctoral Research Scientist in the Columbia Radiation Laboratory, Department of Electrical Engineering, Columbia University. In 1986 he joined the

Hiroshima-Denki Institute of Technology, Hiroshima, Japan, where te is now teaching and pursuing his research interests in avalanche photodetection, quantum electronics, and point processes.

Dr. Matsuo is a member of Sigma Xi.

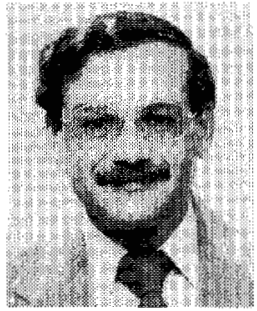

Bahaa E. A. Saleh (M'73-SM'86) received the B.S. degree from Cairo University in 1966 and the Ph.D. degree from the Johns Hopkins University in 1971, both in electrical engineering.

From 1971 to 1974 he was an Assistant Professor at the University of Santa Catarina, Brazil. Thereafter, he joined the Max Planck Institute in Göttingen, Germany, where he was involved in research in laser light scattering and photon correlation spectroscopy. He is presently Professor of Electrical and Computer Engineering at the University of Wisconsin, Madison, where he has been since 1977. He held visiting appointments at the University of California, Berkeley, in 1977 , and the Columbia Radiation Laboratory of Columbia University in 1983. $\mathrm{He}$ is currently involved in research in image processing, optical information processing, statistical optics, optical communication, and vision. $\mathrm{He}$ is the author of Photoelectron Statistics (Springer, 1978) and a co-editor of Transformations in Optical Signal Processing (SPIE, 1981). In 19801983 he was an associate editor of the Journal of the Optical Society of America, and since 1983 he has been a topical editor of the same journal.

Dr. Saleh is a Fellow of the Optical Society of America and a member of Phi Beta Kappa and Sigma Xi. He was appointed a Fellow of the John Simon Guggenheim Foundation in 1984. 